Andreas Böhn - Das zeitgenössische deutschsprachige Sonett 


\author{
Andreas Böhn
}

\title{
Das zeitgenössische deutschsprachige Sonett
}

Vielfalt und Aktualität einer literarischen Form

Verlag J. B. Metzler

Stuttgart - Weimar 
Die Deutsche Bibliothek - CIP-Einheitsaufnahme

Böhn, Andreas:

Das zeitgenössische deutschsprachige Sonett : Vielfalt und Aktualität einer literarischen Form / Andreas Böhn. - Stuttgart ; Weimar : Metzler, 1999

(M-\&-P-Schriftenreihe für Wissenschaft und Forschung)

ISBN 978-3-476-45220-7

ISBN 978-3-476-45220-7

ISBN 978-3-476-04313-9 (eBook)

DOI 10.1007/978-3-476-04313-9

Dieses Werk ist einschließlich aller seiner Teile geschützt. Jede Verwertung außerhalb der engen Grenzen des Urheberrechtsgesetzes ist ohne Zustimmung des Verlages unzulässig und strafbar. Das gilt insbesondere für die Vervielfältigungen, Übersetzung, Mikroverfilmungen und Einspeicherung in elektronischen Systemen.

M \& P Schriftenreihe für Wissenschaft und Forschung

(C) 1999 Springer-Verlag GmbH Deutschland

Ursprünglich erschienen bei J.B. Metzlersche Verlagsbuchhandlung und Carl Ernst Poeschel Verlag GmbH in Stuttgart 1999 


\section{Inhalt}

Vorbemerkung $\ldots \ldots \ldots \ldots \ldots \ldots \ldots \ldots \ldots \ldots \ldots \ldots \ldots \ldots$

I. Das Sonett als problematische Form:

Die neuere Forschungsgeschichte $\ldots \ldots \ldots \ldots \ldots \ldots$

II. Kleine Geschichte des deutschsprachigen Sonetts seit $1945 \quad \ldots \quad 15$

III. Formen als kommunikativer Fundus:

Gebrauch von Formen, Verweis auf Formen . . . . . . . 49

IV. Analysen $\ldots \ldots \ldots \ldots \ldots \ldots \ldots \ldots \ldots \ldots \ldots \ldots \ldots \ldots$

1. Das Sonett als Form des Widerstands und politisches

Vehikel: Von der inneren Emigration bis zu Grass'

,Novemberland' . . . . . . . . . . . . . . . 61

2. Aktualisierung in konservativem Umfeld: ,Swing-Sonette' 76

3. Das Sonett als Verständigungstext: Die DDR-Tradition .. 86

4. Sonett als Spiel-Form: Oskar Pastior . . . . . . . . . . . 99

5. Formaler Konservatismus und thematische Aktualität:

Ludwig Harig und Klaus Modick . . . . . . . . . 113

6. Form-Kontamination und Formzitat . . . . . . . . . . 127

V. Schluß ......................... 137

Literaturverzeichnis . . . . . . . . . . . . . . . . . . . 140

Personenindex ......................... 147 


\section{Vorbemerkung}

Das Sonett dürfte nach wie vor die bekannteste Gedichtform sein, die auch von einem breiteren Publikum als solche erkannt und benannt werden kann. Die markante und einprägsame Form, ja prägnante Formgebung überhaupt verbindet man mit dem Wort, Sonett'. Das wird wohl auch Werbestrategen dazu gebracht haben, es in den sechziger Jahren als Namen für einen Sportwagen zu verwenden, über den es in einem Prospekt hieß: "Poetry in motion - Sonett, a Swedish Saab, different from all others. ${ }^{11}$ Doch taugt das Sonett heute noch für mehr als für solche Reminiszenzen? Ist es nur noch ein Exponat im imaginären Museum der Postmoderne, in dem die Erzeugnisse vergangener Kulturphasen verfügbar gehalten werden und aus dem gerade die Werbung ja auch sonst gerne Versatzstücke der ehemaligen Hochkultur als Material bezieht? Hat die Form ihre Geschichte also endgültig hinter sich und ist zum stereotypen Abziehbild erstarrt, oder wird sie auch noch produktiv, ja gar innovativ verwendet, in einer tatsächlichen Weiterführung der Formentwicklung?

Während die Sonettistik der unmittelbaren Nachkriegszeit großenteils als epigonal abgetan wurde und die Form seit den sechziger Jahren vollends als ,erledigt' galt, findet man in den letzten zwanzig Jahren eine erstaunliche Menge und Vielfalt von Verwendungen des Sonetts. $\mathrm{Ob}$ und in welchem Sinne es sich hierbei um ,richtige' Sonette handelt, erscheint angesichts der zeitgenössischen Produktion als nachrangige Frage (die dennoch nicht vernachlässigt werden soll). Wichtiger ist es zunächst einmal, den Befund festzuhalten, wie viele variantenreiche Kombinationen des Aufgreifens und Gebrauchens der Form mit spielerischen, ironischen, parodistischen Distanznahmen und Verweisen auf

${ }^{1}$ Michael Peterka: Saab. Modelle 92, 93, 95, 96, 99; Sonett I, II und III; 194770; eine Dokumentation. Suderburg 1993, S. 71; vgl. auch S. 89: „Für damalige Verhältnisse galt die Formgebung des Sonett III als letzter Schrei." 
die Form vorliegen. Sie stammen keineswegs nur von Traditionalisten, sondern von Autoren unterschiedlichster Couleur wie Günter Grass, Wolf Biermann, Wolf Wondratschek, Ulla Hahn, Rainer Kirsch, Thomas Rosenlöcher, Gerhard Rühm, Oskar Pastior, Ludwig Harig, Klaus Modick, Ernst Jandl und Durs Grünbein. Entsprechend komplex und heterogen sind die Verwendungsweisen der Form, so daß sie sich kaum noch im Sinne einer Formentwicklung historisch darstellen lassen, sondern methodisch nach der Art und Weise ihrer jeweiligen Funktionalisierung der Form befragt werden müssen. Voraussetzung dafür ist ein Konzept der Zugriffsstrategien auf das Formeninventar, das sich als kommunikativer Fundus verstehen läßt. Solche Strategien sind unter dem Schlagwort ,Intertextualität' ja in erster Linie hinsichtlich der Bezugnahmen auf Einzeltexte breit diskutiert worden, die Bezugnahmen auf Gattungs- und Formtraditionen, vor allem die indirekten, gebrochenen, verdienen aber noch weitaus mehr Interesse, als ihnen bisher zugekommen ist.

Die erstaunliche Renaissance des Sonetts in den letzten beiden Jahrzehnten hat sicher etwas mit der vielerorts bemerkten, Wiederkehr der Formen' zu tun, die sich nach der Abwendung von allen tradierten Formen und Konventionen in den sechziger Jahren in einem erneuten Aufgreifen gerade sehr elaborierter und, altmeisterlicher' Muster seit den Siebzigern manifestiert. Doch gerade diese besonders ,strenge Form scheint, wenn man sich ihr erst einmal zugewendet hat, eine spezifische Sogwirkung zu entfalten. Was auf den Laien schwierig und abschreckend wirkt, kann offensichtlich mit etwas Übung zur täglichen Gewohnheit, wenn nicht zur manischen Besessenheit werden. Das Sonettieren führt gerade bei weniger professionellen Autoren leicht zur Massenproduktion. An Beispielen aus dem deutschen Sprachraum wäre Wilhelm von Humboldts diaristisches Sonettschreiben zu nennen. Auch zeitgenössische Autoren wie Robert Wohlleben oder Klaus M. Rarisch 
kommen auf beachtliche Mengen an Sonetten. Bei letzterem hat diese Fixierung auch eine interpretatorisch-detektivische Seite:

Obwohl von dem Dramatiker Christian Dietrich Grabbe keine Gedichte bekannt sind, hat er doch eine tief verborgene, esoterische Beziehung zur 14zeiligen Sonettform. Wenn man seine beiden Vornamen in der gebräuchlichen Weise abkürzt:

CHR. DIETR. GRABBE

- besteht sein Name aus 14 Buchstaben. Sein bekanntestes und heute noch gespieltes Stück ist

SCHERZ,/SA/TI/RE,/I/RO/NIE/UND/TIE/FE/RE/BE/DEU/TUNG

- ein Dramentitel, der aus 14 Silben besteht. Das Drama ist folgendermaBen gegliedert:

Akt I $=4$ Szenen,

Akt II $=4$ Szenen,

Akt III $=6$ Szenen

- also summa summarum 14 Szenen. Zufall? Oder nicht doch: tiefere Bedeutung? ${ }^{2}$

In solche Tiefen möchte die folgende Untersuchung nicht vordringen. Aber es soll sehr wohl versucht werden, die Verwendungsweisen der Form in ihrer ganzen Breite auszuloten und auch die indirekten Bezugnahmen auf die Sonetttradition angemessen zu berücksichtigen. Statt scheinbar offensichtlich die Form nicht oder nicht vollständig realisierende Texte auszuschließen, wird auch bei ihnen den Verweisen auf das Sonett und ihrer Funktionalisierung nachgegangen. Nur so sind gewisse im einzelnen zu beschreibende Einseitigkeiten und Voreingenommenheiten der bisherigen Forschung zu überwinden, die zu der weitgehenden Mißachtung der Sonettproduktion der achtziger und neunziger Jahre beigetragen haben. Dieser Zeitraum soll daher bei den Analysen im Vordergrund stehen, die auf einem methodischen Kapitel zu Strategien der Formverwendung aufbauen. Dabei wird sich $u$. a. zeigen, daß manche Funktionen historisch sehr stabil sind, andere deutliche Kon-

${ }^{2}$ Klaus M. Rarisch: Die Geigerzähler hören auf zu ticken. Neunundneunzig Sonette mit einem Selbstkommentar. Hamburg 1990, S. $130 f$. 
junkturen haben oder Ausdruck einer ganz spezifischen literarhistorischen Situation sind. Ein vorangehender historischer Überblick bietet eine Vororientierung, die die Einordnung der eingehender analysierten Phänomene erleichtern soll.

Die Entstehung des vorliegenden Bandes wurde wesentlich durch ein Symposion angeregt, das 1997 an der Universität Mannheim stattfand und zu dem der Tagungsband jetzt zeitgleich erscheint. ${ }^{3}$ Den Veranstaltern Theo Stemmler und Stefan Horlacher möchte ich an dieser Stelle herzlich danken, ebenso Julia Bohnengel für kritische Lektüre des Manuskripts und Christoph Weiß für technische Unterstützung.

${ }^{3}$ Erscheinungsformen des Sonetts. Hg. v. Theo Stemmler und Stefan Horlacher. Tübingen 1999. 